\title{
Absence of Positive Results for Flexible Assertive Community Treatment. What is the next Approach?
}

\author{
Tommy Nordén ${ }^{1}$ and Torsten Norlander ${ }^{1,2, *}$
}

\author{
${ }^{I}$ Center for Research and Development, Evidens University College, Göteborg, Sweden; ${ }^{2}$ Department of Clinical Neuro- \\ science, Karolinska Institutet, Solna, Sweden
}

\begin{abstract}
Aims were to review results of the five psychiatric studies on Flexible Assertive Community Treatment (FACT) published during 2007-2013, and to compare FACT with Resource-group Assertive Community Treatment (RACT) which specifically focuses on empowerment and rehabilitation of clients in the stable phase. During 2007 articles appeared in scientific journals arguing in favor of the need for the development of the treatment method Assertive Community Treatment (ACT). A particularly notable article was one that featured a Dutch version of ACT, namely FACT. The initiative received great sympathy given that clinical practice and research showed that both American and British versions of ACT were in need of new impulses to be able to maintain an optimal level of care. Seven years have passed since the Dutch model was international presented and five empirical studies about FACT have been published and therefore a first critical examination of FACT was conducted. The review indicated that the five empirical studies failed to show that FACT involves improvement of the clients in terms of symptoms, functioning, or well-being. The conclusions were that at present there is no evidence for FACT and that RACT with its small, flexible ACT teams, where the client $\mathrm{him} /$ herself is included and decides on the treatment goals, might be able to provide new impulses and a new vitality to the treatment mode of an assertive community treatment.
\end{abstract}

Keywords: Assertive community treatment, assertive outreach, FACT, optimal treatment, RACT, resource group.

\section{INTRODUCTION}

Assertive Community Treatment (ACT) is a program for rehabilitation developed during the 1970's and 1980's which primarily focuses on individuals with long term illness who also require a great deal of psychiatric inpatient care [1]. An ACT-team is a multi-disciplinary team founded on intensive "clinical case management" which primarily is targeting treatment. The efforts are made close to the patient instead of at a clinic or institution. On such a team [2] there must exist a minimum of one psychiatrist, one or two nurses, staff with specific expertise knowledge of drug abuse and dependence, as well as staff with specific competence within work rehabilitation. In total some 10 to 12 professional team members are included who are jointly responsible for approximately 100-120 users. The particular chief variety of an ACT team might be labeled the orthodox ACT models.

The orthodox models can provide evidence based interventions from studies in the USA [3], but further investigations concerning the effectiveness of the models and the possibilities of their applications in different cultural settings should be performed. Studies of ACT in Western Europe do not evidence equally good effects [4], a fact that may be explained by differences in treatment as usual of the treatment and care given in the control groups. In Western Europe normal care was more based on a social-psychiatric paradigm compared to that of the USA, which in turn made

\footnotetext{
*Address correspondence to this author at the Evidens University College, Packhusplatsen 2, SE-411 13 Göteborg, Sweden; Tel: +46 7066211 89; Fax: +463171104 42; E-mail: at.norlander@mailbox.swipnet.se
}

it necessary to further develop ACT with a more comprehensive Assertive Outreach (AO), such as crisis management and the visit of teams in the clients' homes. All in all, however, the British model of ACT (Assertive Outreach Team, AOT) showed high program fidelity to the American orthodox models [5].

In 2007 three different articles were published in scientific journals arguing in different ways for the need for the development of the treatment method Assertive Community Treatment (ACT). The author of the first article was Firn [6] entitled Assertive outreach: has the tide turned against the approach? In this article he discusses how ACT in combination with Assertive Outreach has been successfully implemented in the USA and later was disseminated in Western Europe, in particular in Great Britain where the method was seen by the politicians as a way of providing clients with severe mental illness (SMI) good care and support. But according to Firn, the approach is threatened by shortsighted efforts to save money and focus on traditional community-based teams. Firn [6] argued that in England there is a process where experienced AO teams (AOT) were dissolved and integrated into the community mental health teams (CMHTs). Firn worried that the development might lead to a worsening of the service in various ways and a comprehensive study which compared AO services with CMTHs during 1999-2004 and an 18 month follow up could be summarized as follows, "The benefits of ACT are no greater than with normal community care but patients prefer it" [7]. Firn then referred the reader to qualitative studies indicating that the clients felt that the $\mathrm{AO}$ helping relationships were perceived as normal friendships in comparison to 
the situation within normal care. The clients valued this fact very highly in addition to the efforts to improve the clients self-worth and participation in mainstream activities.

The second article on ACT which received significant attention in the year 2007 was authored by van Veldhuizen and entitled FACT: A Dutch version of ACT [8]. The Dutch model is called Flexible ACT (FACT) and sometimes Function ACT. Flexible (or Function) ACT team is described as a case management team which for "stable long-term patients provides coordinated multidisciplinary treatment and care by individual case management" [8]. However, clients who are in an unstable phase with the risk of suffering a relapse are given shared intensive case management and assertive outreach care by the same team.

The client in the stable group receives a visit by a case manager 2-4 times per month and the client is presented with an individual treatment plan "which is renewed at least once a year and is formulated in a way that patients and their families can understand" [8]. Furthermore, regular visits with "the psychiatrist (medication management, evaluation) and the psychologist (psycho-education, cognitive behavior therapy) take place at the FACT center. Family interventions and supported employment may be added to this plan" [8]. Care for the unstable group is organized around the team's whiteboard. If a client is in danger of falling into an acute condition, his/her name is written on the board and the result is that he/she will be offered ACT or assertive outreach. "The case manager informs the patient (and if necessary the family) that a more intensive care will be organized and that colleagues from the FACT team will work together to prevent readmission and to shorten the crisis" [8]. The client will then meet with a psychiatrist within two days. The FACT team discusses the issue, decides on efforts, and makes a home visit.

The third article which in 2007 discussed the need for development and renewal of ACT was entitled Should we adopt the Dutch version of ACT? "Commentary on FACT: A Dutch version of $A C T$ ?" [9]. The article, by Bond and Drake, appeared in the immediate connection with the Veldhuizen article. The authors discussed the thoughts put forward in the previous article and made the point that several aspects within orthodox ACT need scrutiny and a reexamination. The orthodox models with their large teams with around the clock staffing have had trouble adjusting to more rural contexts where problems with continuity and travel distances are involved. Along with the increased knowledge on investing in early intervention actions regarding mental illness it becomes ever more important thoroughly to examine the target groups of ACT. Perhaps the service provided should not exclusively focus on those most ill but also be used with a broader spectrum of care users.

The most important issue raised by Bond and Drake is perhaps what services should be available to clients who most typically are stable? They argued that the literature is astonishingly unclear as to the vast majority who are not in need of intensive services and that evidence-based guidelines are highly desirable. One of the worries of Bond and Drake regarding FACT is that the method does not specify what case management methodology ought to look like for care users not in crisis: "If usual case management services de- volve to brief contacts with clients, FACT begins to look much like the episodic treatment model in which the case management responds to crises while not fully responding to the needs of most clients" [9]. Bond and Drake conclude their point by raising the notion that scientific questions should be resolved by data rather than by opinions and that both of them as clinical researchers are "calling for empirical examination of assumptions rather than protecting conventional clinical wisdom" [9].

It has now been seven years since van Veldhuizen's article and the current authors have found five empirical articles dedicated to evaluating different aspects of FACT available in international journals. Four of these show data from Holland and one from Great Britain. Thus the time has arrived for the scientific community to do a first critical examination of FACT. The purpose of the current study was to do a brief review of the results and to compare FACT with a successful evidence based ACT method, Resource-group Assertive Community Treatment (RACT). This method is specifically focused on empowerment and rehabilitation of clients in the stable phase.

\section{THE FIVE EMPIRICAL FACT STUDIES}

\section{The First Empirical FACT Article}

The first empirical FACT article was published in 2007 by Bak, van Os, Delespaul, de Bie, a' Campo, Poddighe, and Drukker [10] could be regarded as an explorative study and a starting point for future investigations. It is a pre-post study based on two separate cohorts, pre $(n=116)$ and post $(n=$ 38 ), respectively. Thus, it was not the same people who participated pre and post. In the abstract of the article the authors argued that the proportion of clients who made the transition to remission increased from $19 \%$ during the period before FACT to $31 \%$ during the period after FACT had been implemented in a specific geographic sampling area in Holland. But the main result was not statistically significant! Furthermore, it is not clear which patients who were truly in remission, given that the criteria from the international remission working group were not followed. Of course the recommendations concerning criteria for certain tests were followed, but not as concerns the validity of the criteria which are to last for six months in order to be defined as remission. The study has striking methodological weaknesses which, according to the authors themselves meant that "results consequently were statistically imprecise because of lack of power".

\section{The Second Empirical FACT Article}

The Dutch research group published the second empirical study in 2008 [11]. The study had one FACT group and one non-FACT group. 240 clients receiving FACT were matched with 200 non-FACT clients. Once again there was no significant main effect showing that FACT leads to an increase in remission. However, follow-up measures showed that FACT led to an increase in remission if the patients had indicated on a base line test that they had an unfulfilled need for care with respect to psychotic symptoms. This result is however difficult to interpret and must, as the authors themselves indicated in the Discussion, be dealt with caution. Once again, there remain questions as concerns the concept 
of remission, given that the authors did not follow the criterion of low symptom levels for at least 6 months.

\section{The Third Empirical FACT Article}

In 2011 the research group returned with a third article about FACT [12]. The study used a FACT group $(n=114)$ and a CAU (Care As Usual) group $(n=330)$. The principal questions posed were whether FACT is more cost efficient than CAU and if the pattern of service is changed following the introduction of FACT. It was found that FACT patients more frequently received service as outpatients than did clients with CAU. With respect to cost effectiveness, it was found that costs were $€ 8000$ higher in FACT than in CAU during a 5-year period. In addition there was a decrease in functioning (GAF) in FACT compared with CAU. This latter result further emphasizes that there is no evidence that FACT contributes to increased remission.

\section{The Fourth Empirical FACT Article}

The fourth and most recent article about FACT from the Dutch group [13], as far as we know, is a study from 2013 using secondary data. From earlier collected data and psychiatric case registers, together consisting of six-hundred forty-five individuals, patients who never received FACT, patients ever in FACT (patients who have encountered FACT occasionally), and patients in FACT were identified. The only substantial result in the study was that despite the principle of continuity of care, FACT episodes and nonFACT episodes alternate in a substantial part of the FACT case load. The results indicating that current FACT clients received less inpatient and more outpatient care as well as higher levels of psychosocial functioning than ever-FACT patients and non-FACT clients, must be viewed, at best, as preliminary given that the design of the study and several serious limitations make it impossible to draw causal inferences. Examples of such limitations are that (a) groups may have been different from one another at base line, a fact not examined (!), (b) FACT as a whole was evaluated since authors did not differentiate between the $20 \%$ most severely ill patients and the rest with less intensive treatment, (c) nonFACT clients were older than current-FACT clients and (d) several dependent variable assessments were not actual assessments but were extrapolations.

\section{The Fifth Empirical FACT Article}

The fifth empirical FACT study is a British study [14] with Firn, one of the veterans in terms of applying assertive outreach in the UK, as first author. The study consisted of pre-post assessments in connection with an organizational change. Two ACT teams with assertive outreach (AOT) were to be integrated into the standard care and converted into six standard care "community health teams" (CMHT). The new teams would then function in accordance with the FACT model. The following plan was made up with two levels of intensity: (a) individual case management for a majority of clients in accordance with the standard practice in CMHT, (b) when the patient worsens (a minority of the patients) AO is used within the frame of FACT and the resources from the entire team. The patients were followed up after 12 months. The results presented in the Abstract were as follow: "results show that AO patients $(n=112)$ transferred to standard CMHTs with FACT had significantly fewer admissions and a $50 \%$ reduction of bed use $(21$ fewer admissions and 2,394 fewer occupied bed days) while also receiving a less intensive service (2,979 fewer visits)" [14].

The results are interesting but there is some lack of clarity, and the results presented in the conclusions require critical scrutiny. The major objection is that it is unclear what the pre-post assessment actually measures. As concerns the pre assessment, Firn himself pointed out in his article from 2007 [6] that the assumptions regarding AOT have changed in the UK in comparison to the original assumptions. For this reason it is hazardous to make the point that it was a functioning AOT approach that was integrated into the CMHT. The same kind of lack of clarity pertains to the post condition. The authors themselves argued that "the FACT model implemented in our localities is not equivalent to FACT teams in the Netherlands, which are subject to a fidelity and certification process" [14]. In other words it is not made clear how much assertive outreach was involved in the pre assessments, how much FACT was involved in the post assessments, or how much standard care was included in the two conditions. The lack of clarity of the design makes it necessary to interpret the results with caution. It appears that the chosen model in accordance with the Dutch pattern in fact involved more or less standard care for the majority of the users and intensive case management and assertive outreach for the minority who were clearly very ill. This meant that the researchers took a risk regarding rehabilitation for the majority who were less ill, all in accordance with the fears discussed by Bond and Drake [9]. A measure of this notion might be that the percentage of unemployed clients increased from $67 \%$ pre, to $84 \%$ post.

\section{OVERARCHING COMMENTS ON THE FACT STUD- IES}

Seven years have now passed since FACT was internationally introduced as a new method with a great potential for renewal and further development of Assertive Community Treatment [8]. The initiative was received with great sympathy given that clinical practice and research showed that both the American version of ACT [9] and the British version [7] were in need of new impulses to be able to maintain an optimal level of care. It is unclear whether the same Dutch data set had been used on several occasions, but all in all it is reasonable to assume that at least 750 clients were included in the five empirical FACT studies published in that period. It seems like a sufficient data base on which to make a first evaluation of FACT. We note that the five studies cannot convincingly show that FACT leads to improvement of clients' symptoms, functioning, or well-being. Additional results are meagre, and there exist several methodological limitations. At present there exists no evidence for FACT. In this context it should be noted that our descriptions of the limitations regarding the FACT articles often correspond to what the authors themselves agree on and they should get credit for the transparency in their reports.

We would like to propose the following argument against this background: The original American version of ACT, as well as the further developed British version of AOT, as well 
as the Dutch version of FACT, in essence have the same construction that involves similar limitations. Thus, they can all be seen as variations of the orthodox ACT. What the three models have in common is that they are multidisciplinary and large. It is worth noting that the British AOT originally had high program fidelity in relation to traditional ACT [5] and in terms of FACT there exists information of acceptable fidelity to ACT guidelines [11]. A systematic review [15] with 64 trials including 7,819 clients indicated that ordinary community mental health teams delivered equal outcomes as compared to ACT teams and therefore the conclusion was that the value of investing in high fidelity ACT teams must be in doubt. At the same time ACT, AOT and FACT are surprisingly enough not focused on providing service and treatment to the majority of clients who are largely in a stable phase. As Bond and Drake [9] pointed out there ought to exist evidence based guidelines for how rehabilitation can occur in the best possible way. Against this background we propose that clinicians and theorists within the area of Assertive Community Treatment may show an interest in an evidence based variation of ACT, a variation which places its emphasis on empowerment and rehabilitation of the client, and where the team usually consists of the client him/herself, a physician, a case manager, and perhaps 3 or 4 additional people whom the client chooses. Often a few relatives or friends are included. We refer to Resource group ACT (RACT). This variety is thus either large or multidisciplinary but focuses instead on networking and psychoeducation along with the client and a co-ordination of several different efforts by the standard care.

\section{THE OPTIMAL TREATMENT APPROACH}

It is the version of the assertive outreach and intensive case management which derived from the work of Ian Falloon [16], which most radically has focused on decision making and reinforcement of the client's own selfconfidence and ability (empowerment). The idea was that one could combine all the knowledge about the best evidence based methods used in psychiatry and apply them in a care system, Optimal Treatment. Strategies used in Optimal Treatment were multiple: (a) minimizing anti-psychotic medication through more intensive supervision of and a focus on finding the correct medication in order to reduce symptoms, (b) training of the users and those close to them in methods of stress management, (c) efforts of outreach by care and support staff, (d) goal-oriented training to develop social and professional skills, (e) specific pharmacological and/or psychological strategies to handle unforeseen crisis situations.

In the 1990's a large number of studies were conducted within the frame of Optimal Treatment (subsequently named Resource-group Assertive Community Treatment - RACT) from the vantage point of the manual developed by Falloon. One article [17] reported results from 14 smaller studies within the research project, the Optimal Treatment Project. The article summarized measurement results from 11 countries, obtained from 14 clinics. The data collection took place in the 1990's and in the beginning of the 21 st century. All in all some 603 users (366 men and 237 women) were included with diagnoses within the schizophrenia spectrum. The study had three measurement variables, namely Mental Functions
Impairment Scale (symptoms and functioning), Disability Index (perceived stress and disability of the client) and Global Carer Stress (perceived burden on significant others). Following 24 months of treatment using outreach efforts and resource groups (in accordance with the manual of the method), the patients who had been part of the resource group exhibited significant and substantial improvement on all three measurement variables compared to those who had not received treatment according to the manual.

The international research on RACT, conducted during the period 2001- 2011 has been reported in a meta-analysis [18] and contains 17 published studies based on a total of 2263 clients (1291 men and 972 women). A majority of the participants had a diagnosis of some psychosis (1955) and the rest (308) had other psychiatric diagnoses such as delusion, drug abuse, depression, panic syndrome, anxiety, and obsessive compulsive disorder, conduct disorders, and personality or attentional disorders. Six of the studies had an RCT design and eleven of the studies were observational studies (i.e. they either included reference groups or pre-post assessments on the same participants). In the meta-analysis there were three outcome parameters (a) symptoms, (b) functioning and (c) well-being. The variables were assessed with the aid of well-established clinical tests and other quantifiable data. In order for the studies to be comparable and summarized, a statistical technique was used which recomputed the results as effect sizes (Cohen's $d$ ) in which a small effect size is described as $d=0.20$, a medium effect as $d=0.50$, and a large effect size as $d=0.80$ or greater. The meta-analysis yielded significant effects for both the controlled studies and the observational studies: Symptoms $(d=$ 0.5 , and 0.66 , respectively), Functioning $(d=0.93$, and 0.89 , respectively) and Well-being $(d=1.16$, and 0.57 , respectively). It was also shown that RACT is a useful method for all psychiatric diagnoses. The combined measure for the 17 studies and the three outcome parameters was $d=0.80$. The results may be compared with meta-analyses that combine results using other comparable methods [18], where combined effect sizes typically range between 0.20 and 0.50 .

The RACT model was described in detail in a phenomenological study [19] showing that the RACT manual provides tools for the case manager when working together with the client in forming an effective resource group. The manual contains training programs, training protocols, and methods for evaluating outcomes and quality control. An important element for the empowerment of the client is that the client defines his/her own treatment goals and nominates those who will be included in the Resource Group.

\section{FINAL REMARKS}

The decisive difference between RACT and other ACT models is that the client him/herself is included on the ACT team, and that the treatment has as its starting point the needs and wishes of the client [19]. It represents a sharp contrast with the more orthodox models including FACT. It is shown for example in van Veldhuizen's article [8] that when a client is in the stable phase he/she is presented with an individual treatment plan "which is renewed at least once a year and is formulated in ways that patients and their families can understand" [8]. In accordance with the RACT model the 
client him/herself, with the support of the case manager, determines the goals of the treatment. The road to achieve both the long term and short term goals is jointly discussed and decided on in the Resource Group a minimum of four times per year, or even more frequently if called for. The Resource Group is a small ACT team and between meetings different constellations of the Resource Group work on different tasks. Thus the client and a relative might work on certain social skills, or the client may practice stress management with the case manager or another member of the Resource Group. If specific knowledge is needed of e.g. drug abuse or supported employment, an expert may be added to the group.

The small format of the ACT team (i.e., the Resource Group), where the client is the person who decides the goals of treatment, has been shown to be an advantage both in terms of flexibility and degree of integration [19]. At the same time the format does not allow for the use of traditional scales for fidelity assessments for original ACT e.g. the Dartmouth Assertive Community Treatment Fidelity Scale (DACT) [20] in connection with RACT. The Clinical Strategies Implementation scale (CSI) was designed for the measurement of the fidelity of RACT and the scale is now available [21] in a modernized and revised version. Future research on fidelity assessments in connection to different ACT models, and perhaps combinations of models may elucidate which components are most effective and which ones should be changed. Models should of course not be carved in stone, but must constantly evolve in order to keep an optimal treatment.

\section{CONFLICT OF INTEREST}

The authors confirm that this article content has no conflict of interest.

\section{ACKNOWLEDGEMENTS}

Declared none.

\section{REFERENCES}

[1] Stein LL. A historical review of the Madison model of community care assertive community treatment. Hosp Commun Psychiatry 1990; 41: 649-51.

[2] Lewin Group. Assertive Community Treatment literature review. Prepared for health care and financing administration and substance abuse and mental health services administration. Falls Church, VA: The Lewin Group 2000.

[3] Bond GR, Drake RE, Muesser KT, Latimer E. Assertive community treatment for people with severe mental illness, critical ingredients and impacts on patients. Dis Manag Health Outcome 2001; 9: $142-57$.
[4] Burns T, Fioritti A, Holloway F, Malm U, Rössler W. Case management and assertive community treatment in Europe. Psychiatr Serv 2001; 52: 631-6.

[5] Fiander M, Burns T, McHugo GJ, Drake, RE. Assertive community treatment across the Atlantic: comparison of model fidelity in the UK and USA. Br J Psychiatry 2003; 182: 248-54.

[6] Firn M. Assertive outreach: has the tide turned against the approach? Ment Health Prac 2007; 10: 24-7.

[7] Killaspy H, Bebbington P, Blizard R. The REACT study: randomized evaluation of assertive community treatment in north London. Br Med J 2006; 332: 815-8.

[8] van Veldhuizen JR. FACT: a Dutch version of ACT. Commun Ment Health J 2007; 43: 421-33.

[9] Bond GR, Drake RE. Should we adopt the Dutch version of ACT? Commentary on "FACT: a Dutch version of ACT". Commun Ment Health J 2007; 43: 435-8.

[10] Bak M, van Os J, Delespaul P, et al. An observational, "real life", trial of the introduction of assertive community treatment in a geographically defined area using clinical rather than service use outcome criteria. Soc Psychiatry Psychiatr Epidemiol 2007; 42: 12530 .

[11] Drukker M, Maarschalkerweerd M, Bak M, et al. A real-life observational study of the effectiveness of FACT in a Dutch mental health region. BMC Psychiatry 2008; 8: 93 doi: 10.1186/1471244X-8-93

[12] Drukker M, van Os J, Sytema S, Driessen G, Visser E, Delespaul P. Function assertive community treatment (FACT) and psychiatric service use in patients diagnosed with severe mental illness. Epidemiol Psychiatr Sci 2011; 20: 273-8.

[13] Drukker M, Visser E, Sytema S, van Os J. Flexible Assertive Community Treatment: Severity of symptoms and psychiatric health service use, a real life observational study. Clin Pract Epidemiol Ment Health 2013; 9: 202-9.

[14] Firn M, Hindhaugh K, Hubbeling D, Davies G, Jones B, White SJ. A dismantling study of assertive outreach services: comparing activity and outcomes following replacement with the FACT model. Soc Psychiatry Psychiatr Epidemiol 2013; 48: 997-1003.

[15] Burns T. The rise and fall of assertive community treatment? Int Rev Psychiatry 2010; 22; 130-7.

[16] Falloon IHR. Optimal Treatment for psychosis in an international multisite demonstration project. Psychiatr Serv 1999; 615-8.

[17] Falloon I, Montero I, Sungur M, et al. Implementation of evidencebased treatment for schizophrenic disorders: two-year outcome of an international field trial of Optimal Treatment. World Psychiatry 2004; 3: 104-9.

[18] Nordén T, Malm U, Norlander T. Resource group Assertive Community Treatment (RACT) as a tool of empowerment for clients with severe mental illness: A meta-analysis. Clin Pract Epidemiol Ment Health 2012; 8: 144-51.

[19] Nordén T, Eriksson A, Kjellgren A, Norlander T. Involving clients and their relatives and friends in the psychiatric care. Case managers' experiences of training in Resource group Assertive Community Treatment. Psych J 2012; 1: 15-27.

[20] Bond GR, Salyers MP. Prediction of Outcome from the Dartmouth Assertive Community Treatment Fidelity Scale, CNS Spect 2004; 9: 937-42.

[21] Andersson J, Ivarsson B, Tungström S, Malm, U, Norlander, T. The Clinical Strategies Implementation scale Revised (CSI-R). Fidelity assessment of Resource group Assertive Community Treatment. Open J Med Psychol 2014; 3: 36-41. 